\title{
Serum Anti-CCNY Autoantibody Is an Independent Prognosis Indicator for Postoperative Patients with Early-Stage Nonsmall-Cell Lung Carcinoma
}

\author{
Li Ma, Wentao Yue, Yu Teng, Lina Zhang, Meng Gu, and Yue Wang \\ Department of Cellular \& Molecular Biology, Beijing TB and Thoracic Tumor Research Institution/Beijing Chest Hospital, \\ Capital Medical University, 97 Beimachang, Tongzhou, Beijing 101149, China
}

Correspondence should be addressed to Wentao Yue; yuewt2000@yahoo.com

Received 15 June 2013; Revised 20 August 2013; Accepted 21 August 2013

Academic Editor: Sun-Il Hwang

Copyright (C) $2013 \mathrm{Li} \mathrm{Ma}$ et al. This is an open access article distributed under the Creative Commons Attribution License, which permits unrestricted use, distribution, and reproduction in any medium, provided the original work is properly cited.

Cyclin Y (CCNY) is a novel cyclin and almost nothing is known about its role in human cancers. To investigate the clinical significance of serum anti-CCNY autoantibodies in nonsmall-cell lung carcinoma (NSCLC), the serum levels of CCNY protein in 264 patients with NSCLC, 103 patients with tuberculosis, and 89 healthy controls were analyzed by immunohistochemistry. The result shows that, compared with normal lung tissues, the NSCLC tissues contained higher levels of CCNY protein. The levels of anti-CCNY autoantibodies were higher in the sera of the patients with NSCLC than in the sera of the healthy controls $(P<0.001)$ or the patients with tuberculosis $(P=0.027)$. Moreover, in a Cox regression analysis, anti-CCNY autoantibody was an independent factor that predicted poor prognosis for postoperative patients with early-stage NSCLC $(P=0.026)$ as well as for those with distant metastasis $(P=0.012)$. Our data indicated that Anti-CCNY autoantibody may be useful as a latent tumor marker to facilitate diagnosis and may represent a novel prognostic indicator for patients with early stage NSCLC.

\section{Background}

In recent decades, the incidence of lung cancer has decreased in some countries although lung cancer remains the leading cause of cancer-related mortality worldwide [1-5]. Nonsmall cell lung cancer (NSCLC) accounts for $75 \%$ to $80 \%$ of all cases of lung cancer. Surgical treatment, chemotherapy, targeted therapy, and radiotherapy are the usual therapeutic approaches used for NSCLC patients. In particular, surgery is the preferred method in the treatment of patients with clinical stages I-IIIA NSCLC. At present, other than imaging as part of conventional detection, there are no indicators or methods to evaluate therapeutic efficacy, even though prognostic and predictive biomarkers have been widely investigated. Therefore, there is an urgent need for sensitive indicators and effective methods to evaluate treatment efficacy and to assess prognosis.

Cell cycle progression is an important regulatory mechanism for cell proliferation, as dysregulation of the cell cycle results in unrestrained cell proliferation as a consequence of tumorigenesis [6]. Cyclins and cyclin-dependent kinases (CDKs) are important components of the regulation of the cell cycle. Cyclins play important roles in controlling progression through the cell cycle by activating cyclin-dependent kinase enzymes (CDK) [7-9]. Cyclin Y (CCNY) is the latest cell cycle protein to be identified from a human testis cDNA library. CCNY acts as a growth factor sensor, to integrate extracellular signals with the cell cycle machinery [10]. Recent studies have shown that CCNY mRNA is overexpressed in NSCLC tissues [11] and that altered levels of the CCNY protein influence cell proliferation, colony formation, and cell cycle progression in glioma cells [12]. However, the precise biological function and clinical significance of CCNY remain unclear. Therefore, the purpose of the present study was to examine the potential clinical values of CCNY and serum anti-CCNY autoantibodies in patients with NSCLC.

In the current study, we first examined the expression profile of CCNY in tumors from tissues of 39 NSCLC patients by immunohistochemistry. Compared with adjacent nondiseased lung tissues, CCNY was upregulated in lung cancer 
cells. We then investigated the CCNY autoantibody expression in circulating serum from 264 patients with NSCLC, 103 patients with tuberculosis, and 89 healthy controls. Compared to the levels in healthy individuals and patients with tuberculosis, the CCNY autoantibody level was elevated in NSCLC. Detailed analysis indicated that the anti-CCNY autoantibody was an independent factor that predicted poor prognosis for postoperative patients with early stage NSCLC $(P=0.026)$ as well as for those with distant metastasis $(P=0.012)$, implying its potential practical use as an effective indicator of the clinical prognosis of NSCLC.

\section{Patients and Methods}

2.1. Serum and Samples. Serum samples from 264 patients with NSCLC, 89 age-matched healthy controls, and 103 patients with tuberculosis were obtained from the Beijing Chest Hospital Samples Bank in the period from July 2008 to July 2009. The patients who provided the samples comprised of 186 men and 78 women (median age, 58.5 years; range, 33-85 years). The clinical characteristics of the patients with NSCLC are shown in Table 1. In addition, 39 pairs of lung cancer and paratumorous tissues were obtained at the same time.

The patients' serum samples were collected at initial diagnosis. None of the patients had received preoperative chemotherapy or radiotherapy. The healthy controls were recruited during annual health examinations and they showed no evidence of malignancy. Paratumorous tissues, which were obtained from healthy tissues distant from malignant tumors, were microscopically confirmed by $\mathrm{H} \& \mathrm{E}$ staining to contain normal bronchus and pulmonary alveolus tissue and to lack malignant cells. Tumor histology was classified according to the World Health Organization guidelines for histologic typing of lung tumors. Tumor-nodemetastasis (TNM) stage was defined in accordance with the seventh edition of the TNM Classification of Lung Cancer. For survival analysis, the follow-up period was limited to 3 years. The study protocol was approved by the Beijing Chest Hospital Medical Ethics Committee according to the legal regulations of TNM.

Blood samples were subjected to centrifugation of the supernatants for 15 minutes at $3000 \times \mathrm{g}$ at room temperature and immediately stored at $-80^{\circ} \mathrm{C}$ for further analysis.

2.2. Immunohistochemistry (IHC). Briefly, paraffin-embedded sections of resected specimens were dewaxed, rehydrated, trypsinized, and boiled in $0.01 \mathrm{~mol} / \mathrm{L}$ citrate buffer for $20 \mathrm{~min}$. For immunostaining, the rabbit polyclonal antiCCNY antibody (Abcam, ab80853), which specifically recognizes a peptide that corresponds to amino acids 144-193 of the human CCNY protein, was used at a 1:50 dilution and incubated at $4^{\circ} \mathrm{C}$ for $12 \mathrm{~h}$, followed by incubation with biotinylated goat anti-rabbit IgG and streptavidin horseradish peroxidase. The streptavidin-peroxidase method was used routinely. All slides were examined and scored independently by two pathologists (Yu Teng and Haiqing Zhang) who had no prior knowledge of the clinical data and graded the staining intensity independently in all cases. Based on the percentage
TABLE 1: The characteristics of the NSCLC patients.

\begin{tabular}{|c|c|}
\hline Characteristics & No \\
\hline Total & $264(100 \%)$ \\
\hline \multicolumn{2}{|l|}{ Age } \\
\hline$\leq 60$ & $149(56.44 \%)$ \\
\hline$>60$ & $115(43.56 \%)$ \\
\hline \multicolumn{2}{|l|}{ Gender } \\
\hline Man & $186(70.46 \%)$ \\
\hline Woman & $78(29.54 \%)$ \\
\hline \multicolumn{2}{|l|}{ Smoke } \\
\hline Yes & $156(59.09 \%)$ \\
\hline No & $108(40.91 \%)$ \\
\hline \multicolumn{2}{|l|}{ Smoke index } \\
\hline$\leq 400$ & $46(29.49 \%)$ \\
\hline$>400$ & $110(70.51 \%)$ \\
\hline \multicolumn{2}{|l|}{ Pathology type } \\
\hline S & $130(49.24 \%)$ \\
\hline A & $134(50.76 \%)$ \\
\hline \multicolumn{2}{|l|}{ Tumor site } \\
\hline Right & $115(43.56 \%)$ \\
\hline Left & 149 (56.44\%) \\
\hline \multicolumn{2}{|l|}{ Differentiation } \\
\hline Well + moderate & $161(60.98 \%)$ \\
\hline Poor & $103(39.02 \%)$ \\
\hline \multicolumn{2}{|l|}{ Tumor size } \\
\hline $\mathrm{T} \leq 3 \mathrm{~cm}$ & $130(49.2 \%)$ \\
\hline $\mathrm{T}>3 \mathrm{~cm}$ & $134(50.8 \%)$ \\
\hline \multicolumn{2}{|l|}{ Lymph node stage } \\
\hline No & $124(47.0 \%)$ \\
\hline $\mathrm{N} 1,2,3$ & $140(53.0 \%)$ \\
\hline \multicolumn{2}{|l|}{ Distant metastasis } \\
\hline M0 & $189(71.6 \%)$ \\
\hline M1 & $75(28.4 \%)$ \\
\hline \multicolumn{2}{|l|}{ Clinical stage } \\
\hline I & $74(28.03 \%)$ \\
\hline II & $39(14.77 \%)$ \\
\hline III & $76(28.79 \%)$ \\
\hline IV & $75(28.41 \%)$ \\
\hline
\end{tabular}

S: squamous cell carcinoma.

A: adenocarcinoma.

Define $P<0.05$ as the significant difference.

of positive cells and staining intensity, the results of the CCNY staining were classified as negative $(0 \%)$, weakly positive, moderately positive, and strongly positive. Staining intensity was scored as follows: (1) $\leq 10 \%$ of the tumor area stained, 0 ; (2) $11-25 \%$ stained, 1 ; (3) $26-50 \%$ stained, 2 ; and (4) $\geq 51 \%$ stained, 3 . When stained tumor cells made up more than $25 \%$ of the tumor, the sample was graded as positive.

2.3. Expression and Purification of Recombinant Proteins. The full-length cDNA fragment that encodes CCNY was amplified from A549 cells and inserted into the prokaryotic 
expression vector pET30a. Then, the pET30a-CCNY-6His plasmid was transformed into Escherichia coli BL21 (DE3) and induced with $1 \mathrm{mM}$ IPTG for $4 \mathrm{~h}$ at $37^{\circ} \mathrm{C}$. The CCNY fusion protein, which was mainly expressed in inclusion bodies, was purified and refolded using a Ni-charged IDA agarose affinity chromatography column (Qiagen). Electrophoresis and Western blotting were used to analyze the expression levels of the recombinant proteins. A mouse monoclonal antibody directed against the $6{ }^{*}$ His-tag (ab15149) and a rabbit polyclonal anti-CCNY antibody (ab80853) were provided by Abcam.

2.4. Enzyme-Linked Immunosorbent Assay (ELISA). The CCNY protein was used at $1 \mu \mathrm{g} / \mathrm{mL}$ as the coating antigen, and a standard curve was constructed using the CurveExpert 13.0 software. The detection range of the serum anti-CCNY autoantibody was $25-800 \mathrm{ng} / \mathrm{mL}$. Samples with concentrations that fell outside the linear detection range of the standard curves were rediluted and remeasured.

Microtiter 96-well plates were coated with $100 \mu \mathrm{L}$ per well of $1 \mu \mathrm{g} / \mathrm{mL}$ CCNY protein overnight at $4^{\circ} \mathrm{C}$. The wells were blocked with $5 \% \mathrm{w} / \mathrm{v}$ nonfat milk in PBS-T (PBS $(\mathrm{pH}$ 7.2) that contained $0.05 \% \mathrm{v} / \mathrm{v}$ Tween-20) for $60 \mathrm{~min}$ at $37^{\circ} \mathrm{C}$ and then incubated for $90 \mathrm{~min}$ at $37^{\circ} \mathrm{C}$ with human serum samples diluted 1:100 in PBS. After five washes with PBS$\mathrm{T}$, the samples were incubated with horseradish-peroxidase (HRP-) conjugated goat anti-human IgG (diluted 1:5,000, ab97165; Abcam) as the secondary antibody for $60 \mathrm{~min}$ at $37^{\circ} \mathrm{C}$, followed by five washes with PBS-T. Finally, $100 \mu \mathrm{L}$ of TMB was added to each well, followed by incubation for $15 \mathrm{~min}$ at room temperature. Subsequently, $50 \mu \mathrm{L}$ of $2 \mathrm{M}$ $\mathrm{H}_{2} \mathrm{SO}_{4}$ as the stop solution was added to each well and mixed thoroughly. The optical density (OD) at $450 \mathrm{~nm}$ was read in a spectrophotometer within $30 \mathrm{~min}$.

To ensure that the immunoassay was suitable for measuring clinical serum sample, reproducibility and linearity were examined. The assay showed excellent linearity with serial dilutions, with a $10 \%$ coefficient of variation $(\mathrm{CV})$ for the intra and interassay variabilities.

2.5. SDS-PAGE Electrophoresis and Western Blotting. The purified CCNY protein $(0.1 \mu \mathrm{g})$ of each sample was separated on a $12.5 \%$ homogeneous SDS-PAGE gel and electrotransferred onto a nitrocellulose membrane (NC). The NC membrane was blocked with 5\% w/v nonfat milk in PBS-T (PBS ( $\mathrm{pH}$ 7.2) that contained $0.1 \% \mathrm{v} / \mathrm{v}$ Tween-20). The NC membrane was then cut into small strips and incubated overnight at $4^{\circ} \mathrm{C}$ with anti-CCNY antibody (1:1000 dilution, ab80853; Abcam), anti-His-tag antibody (1:1000 dilution, M089-3; MBL International), six serum samples from patients with NSCLC, and one negative control (1:500 dilutions), respectively. Then, peroxidase-conjugated secondary antibodies (goat anti-mouse IgG, ab97040; goat anti-rabbit IgG, ab7090; goat anti-human IgG, ab97165; all from Abcam) were incubated for $60 \mathrm{~min}$ at $37^{\circ} \mathrm{C}$. Finally, the immunoreactive bands were visualized using the ECL kit (Invitrogen) according to the manufacturer's instructions, and the images were acquired using the Alpha Innotech Digital Imaging System.
2.6. Statistical Analysis. The data were analyzed using the SPSS 16.0 software package. The Wilcoxon signed rank sum test was applied to analyze differences between the paratumorous tissues and tumor tissues. The serum antiCCNY autoantibody concentrations for the various groups (i.e., healthy controls, patients with tuberculosis, and patients with NSCLC with different degrees of differentiation) are presented as median values and ranges. Normality was ensured using the Kolmogorov-Smirnoff test. The Mann-Whitney nonparametric $U$-test was used to evaluate the differences in serum anti-CCNY autoantibody concentrations between these groups. Receiver operating characteristic (ROC) curves were constructed, the area under the curve (AUC) was measured, and the cutoff value was determined from the mean plus two standard deviations derived using the sera of the healthy controls. OS was defined as the time interval between the date of diagnosis and the date of death or the last followup. Survival curves were calculated using the KaplanMeier method, and comparisons between the different groups were analyzed using the log-rank test. A multivariate analysis was performed using the Cox regression method, to identify the prognostic factors for OS from the variables with proven significance in the multivariate analysis. A $P$ value $<0.05$ indicated a statistically significant difference.

\section{Results}

3.1. The CCNY Protein Is Overexpressed in NSCLC Tissues. We measured the expression levels of CCNY protein in 39 pairs of NSCLC tissues and paratumorous tissues by immunohistochemistry. The CCNY protein was found mainly in the plasma and partially localized to the plasma membrane. Positive expression of the CCNY protein was recognized in $41.0 \%(16 / 39)$ and $23.1 \%(9 / 39)$ of the tumor tissues and paratumorous tissues, respectively $(P=0.089)$. The average scores (on a scale of $0-3$ ) for CCNY protein expression were $2.154 \pm 1.368$ and $1.743 \pm 0.965$ for the tumor tissues and paratumorous tissues, respectively $(P<0.001)$. Therefore, CCNY protein expression was significantly higher in the lung cancer tissues than in the paratumorous tissues (Figure 1).

3.2. Construction of the Expression Vector and Purification of the Fusion Protein. The PCR products were separated, extracted, purified, and ligated into the pET30a vector. The recombinant plasmid vector was digested with $\mathrm{NcoI}$ or/and XhoI and run on an agarose gel. The fragment of interest in the recombinant plasmid migrated to the 1000-bp marker, corresponding to the size of the CCNY gene (Figure 2(a)). DNA sequencing showed that the inserted fragment was $1026 \mathrm{bp}$ in length, and the sequence of this fragment completely matched the CCNY nucleotide sequence in GenBank. Thus, the CCNY expression vector was successfully constructed.

The fusion protein was detected in inclusion bodies with an expected distinct band on SDS-PAGE, which was not observed in the negative control sample (Figure 2(b)). This result was further confirmed by Western blotting with the anti-His antibody and anti-CCNY antibody (Figure 2(c)). The molecular mass of the CCNY fusion protein was 


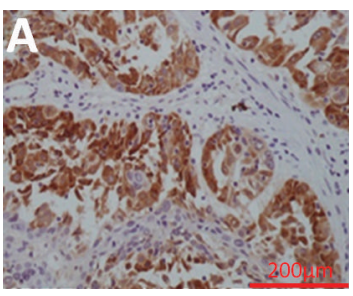

(a)

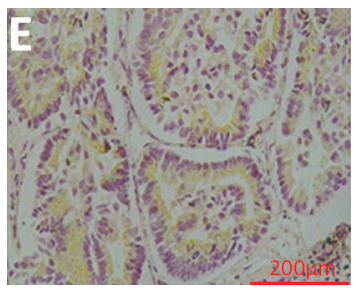

(e)

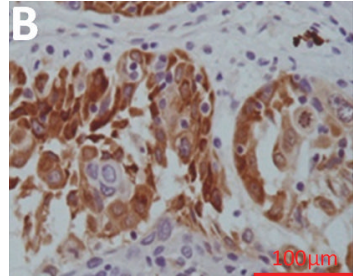

(b)

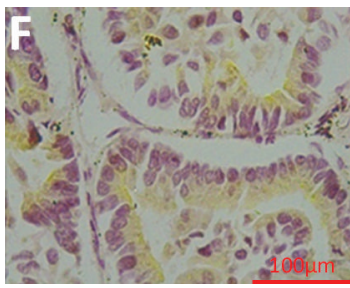

(f)

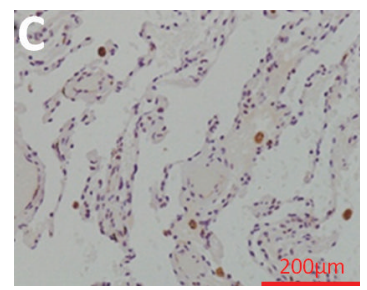

(c)

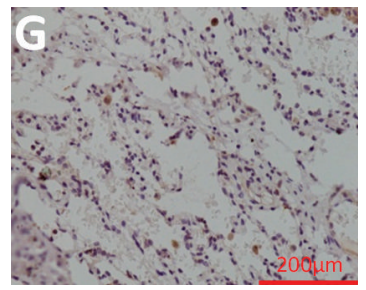

(g)

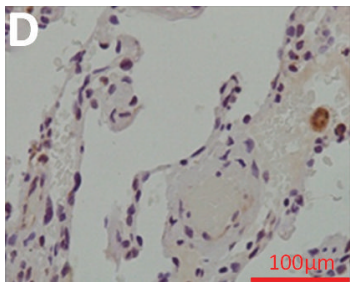

(d)

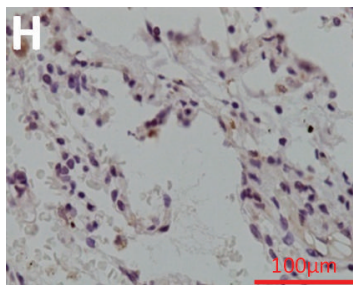

(h)

Figure 1: Analyses of CCNY protein expression using immunohistochemistry. Positive expression of CCNY protein in squamous-cell carcinoma sample ((a): 20x, (b): 40x). Negative expression of CCNY protein in matched para-squamous-cell carcinoma sample ((c): 20x, (d): 40x). Positive expression of CCNY protein in adenocarcinoma sample ((e): 20x, (f): 40x). Negative expression of CCNY protein in matched para-adenocarcinoma sample ((g): 20x, (h): 40x).

approximately $45 \mathrm{kD}$, which is larger than the estimated size of $37 \mathrm{kD}$ owing to the fact that it contains some amino acids encoded by the vector. These results confirmed that the CCNY protein was successfully expressed in E. coli (DE3). The CCNY protein was purified with the Ni2+affinity chromatography purification system using a routine procedure, and high-purity CCNY protein was obtained.

3.3. Increased Levels of Serum Anti-CCNY Autoantibodies in Patients with NSCLC. The serum anti-CCNY autoantibody levels were significantly higher in the patients with NSCLC $(N=264 ;$ median, $76.07 \mathrm{ng} / \mathrm{mL}$; range, $0.01-238.18 \mathrm{ng} / \mathrm{mL})$ than in the healthy controls $(N=89$; median, $51.28 \mathrm{ng} / \mathrm{mL}$; range, $0.01-134.53 \mathrm{ng} / \mathrm{mL} ; P<0.001)$ or in the patients with tuberculosis $(N=103$; median, $68.69 \mathrm{ng} / \mathrm{mL}$; range, $0.01-$ $208.12 \mathrm{ng} / \mathrm{mL} ; P=0.027$ ) (Figure 3(a)). An ROC curve analysis was performed to determine the values that distinguished NSCLC patients from healthy controls. The area under the ROC curve (AUC) was 0.737 (95\% CI, 0.6770.796). A cutoff value of $112.5 \mathrm{ng} / \mathrm{mL}$ was applied to provide optimal diagnostic accuracy (minimize false-negatives), giving a sensitivity of $23.5 \%$ and a specificity of $95.5 \%$ (positive predictive value (PPV), 93.9\%; negative predictive value (NPV), 29.6\%) (Figure 3(b)). No correlation was observed between the rate of anti-CCNY autoantibody positivity and the clinical pathologic variables.

To confirm the results of ELISA test, we selected four serum samples that were detected anti-CCNY autoantibodypositive by ELISA and two serum samples of anti-CCNY autoantibody-negative from the NSCLC group, and one sample was selected from healthy group as blank control. The positive serum samples showed better immunoreactivity, and there was no immunoreactivity in the negative samples and the blank control (Figure 2(c)).
3.4. Relationship between Anti-CCNY Autoantibody Levels and Survival. In total, 213 patients were included in the survival analyses. The median survival time was 23 months and the 3-year survival rate was $39.4 \%$. In all, 125 patients received surgical treatment and 88 patients received chemotherapy. The levels of anti-CCNY autoantibodies were also evaluated for a possible association with OS. The KaplanMeier analysis showed no significant association between the levels of anti-CCNY autoantibodies and OS (for 3-year OS: anti-CCNY autoantibody-positive group (33.3\%) versus antiCCNY autoantibody-negative group (41.2\%); for median OS: anti-CCNY autoantibody-positive group (17 months) versus anti-CCNY autoantibody-negative group (24 months), $P=$ 0.133), (Figure 4(a)). To eliminate the influence of different therapies on OS, we assessed the relationships between the levels of anti-CCNY autoantibody and the OS of patients who received surgical treatment or chemotherapy. We found that the anti-CCNY autoantibody-positive patients had a shorter OS (for 3-year OS: anti-CCNY autoantibody-positive group [39.3\%] versus anti-CCNY autoantibody-negative group [60.8\%]; for median OS: anti-CCNY autoantibody-positive group [22 months] versus anti-CCNY autoantibody-negative group [ $>36$ months], $P=0.014$ ). For the patients with NSCLC who received chemotherapy, the OS rates were not significantly different between the anti-CCNY autoantibodypositive group and anti-CCNY autoantibody-negative group $(P=0.253)$.

3.5. Anti-CCNY Autoantibodies Represent an Independent Risk Factor for Survival in Postoperative Patients with EarlyStage NSCLC. A multivariate analysis was performed using the Cox Backward Wald regression model, to assess the effects on OS of age, gender, smoking, smoking index, tumor site, pathology type, $\mathrm{T}, \mathrm{N}$, and $\mathrm{M}$, the levels of serum anti-CCNY 


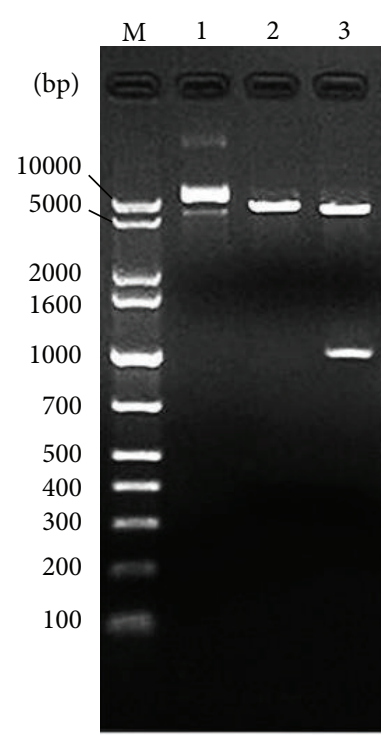

(a)

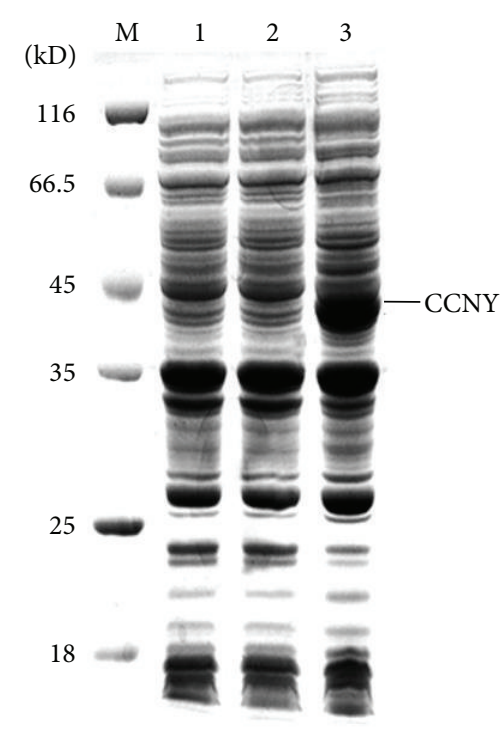

(b)

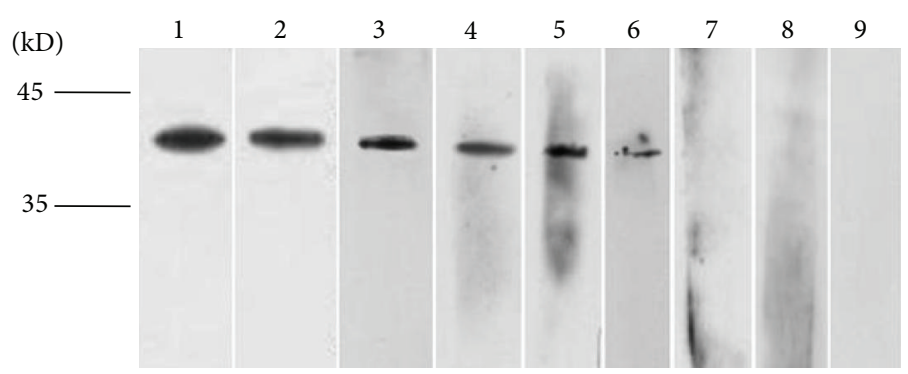

(c)

Figure 2: (a) The recombinant vectors pET30a-CCNY-6His were certificated by restricted enzyme cutting. Lane M: DNA marker; Lane 1: recombinant vectors pET30a-CCNY-6His, Lane 2: NCO I single restriction enzyme digestion, Lanes 3: NCO I and Xho I double restriction enzyme digestion. (b) SDS-PAGE analysis was performed to identify the expression of CCNY protein. Lane M: protein molecular weight marker, Lane 1: the negative control of BL21 (DE3), Lane 2: the negative control of BL21 (DE3)/pET30a, Lane 3: CCNY protein expressed in BL21 (DE3)/pET30a. (c) The validation of CCNY autoantibody. Lane 1: positive control (Rabbit anti-CCNY polyclonal antibody), Lane 2: positive control (His-tag antibody), Lanes 3, 4, 5, and 6: the serum with positive CCNY autoantibody, Lanes 7 and 8: the serum with negative CCNY autoantibody, Lane 9: negative control.

autoantibodies, and differentiation of the tumor. Finally, the levels of serum anti-CCNY autoantibodies and distant metastasis were included in this model. The serum anti-CCNY antibody levels ( $\mathrm{HR}=2.380,95 \% \mathrm{CI} 1.110-5.304, P=0.026)$ and distant metastasis $(\mathrm{HR}=4.092,95 \%$ CI 1.371-12.210, $P=0.012$ ) persisted as independent prognostic factors for patients with NSCLC. The results of the multivariate analysis of survival are summarized in Table 2.

\section{Discussion}

Autoantibodies against tumor-associated antigens are frequently found in the sera of patients with cancer, and they have been analyzed in many previous studies [13-15]. Many studies have described how cyclins A, B, D, and E and their respective autoantibodies are overexpressed in various cancers, including lung cancer $[14,16-21]$. Although CCNY was identified as a novel member of the cyclin family, almost nothing is known about the clinical value of the CCNY protein and anti-CCNY autoantibodies, and only a few studies have reported that the levels of mRNA for CCNY are upregulated in glioma cells and NSCLC tissues [11, 12, 22]. In the present study, we initially used immunohistochemistry to measure the expression levels of CCNY protein in 39 pairs of NSCLC tissues and paratumorous tissues.

It is reported to be a useful approach for cancer detection and diagnosis by detecting autoantibody using recombinant protein $[18,23,24]$. Therefore, we cloned and expressed a CCNY fusion protein in E. coli BL21 (DE3), established an indirect ELISA method, and analyzed the levels of antiCCNY autoantibodies in the sera of 264 patients with NSCLC who had not yet undergone treatment, 103 patients with tuberculosis, and 89 healthy controls.

The results show that the levels of CCNY protein and antiCCNY autoantibody are elevated in patients with NSCLC. The factors that lead to the production of anti-CCNY autoantibody are not completely understood, and we assume that the autoantibody that appears with malignant transformation 


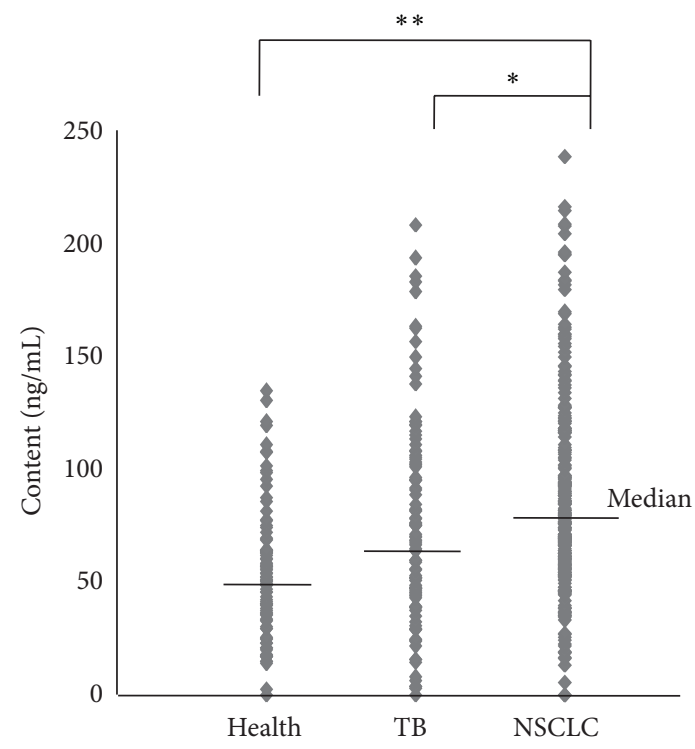

(a)

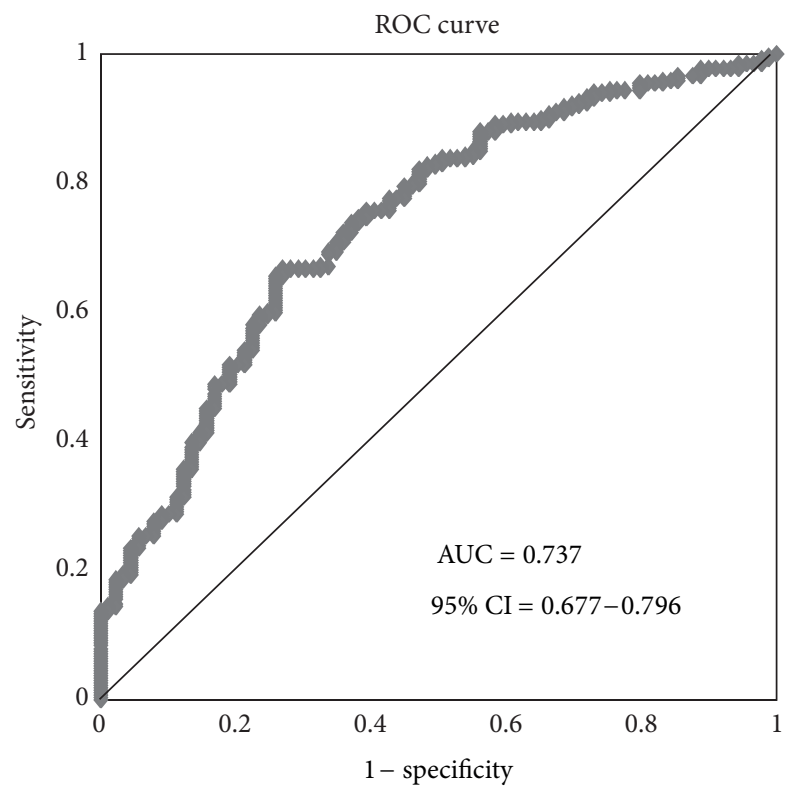

(b)

FIgURE 3: (a) The anti-CCNY antibody level was detected by ELISA for serum samples from each group. (b) An ROC analysis of serum anti-CCNY antibody levels in no small cell lung cancer patients versus healthy controls.

TABLE 2: Multivariate analysis of prognostic factors in patients with NSLCL.

\begin{tabular}{|c|c|c|c|c|}
\hline Covariate & Covariate subgroup & HR & $95 \% \mathrm{CI}$ & $P$ \\
\hline Age & $\begin{array}{l}\leq 60 \\
>60\end{array}$ & 1.127 & $0.557-2.281$ & 0.739 \\
\hline Gender & $\begin{array}{c}\text { Male } \\
\text { Female }\end{array}$ & 1.705 & $0.353-8.241$ & 0.323 \\
\hline Smoke & $\begin{array}{l}\text { Yes } \\
\text { No }\end{array}$ & 0.189 & $0.013-2.713$ & 0.220 \\
\hline Smoke index & $\begin{array}{l}\leq 400 \\
>400\end{array}$ & 0.485 & $0.219-1.072$ & 0.740 \\
\hline Tumor site & $\begin{array}{l}\text { Right } \\
\text { Left }\end{array}$ & 1.522 & $0.712-3.251$ & 0.278 \\
\hline Pathology type & $\begin{array}{l}\text { A } \\
S\end{array}$ & 0.565 & $0.236-1.352$ & 0.200 \\
\hline Differentiation & $\begin{array}{c}\text { Poor } \\
\text { Moderate + well }\end{array}$ & 0.616 & $0.304-1.248$ & 0.179 \\
\hline Levels of CCNY antibody & $\begin{array}{l}\text { Negative } \\
\text { Positive }\end{array}$ & 4.044 & $1.849-8.843$ & 0.006 \\
\hline $\mathrm{T}$ & $\begin{array}{c}\mathrm{T} 1 \\
\mathrm{~T} 2,3,4\end{array}$ & 1.290 & $0.838-1.981$ & 0.247 \\
\hline $\mathrm{N}$ & $\begin{array}{c}\text { N0 } \\
\text { N1, 2, } 3\end{array}$ & 1.121 & $0.763-1.645$ & 0.561 \\
\hline M & $\begin{array}{l}\text { M0 } \\
\text { M1 }\end{array}$ & 4.485 & $1.412-14.243$ & 0.011 \\
\hline Levels of CCNY antibody & $\begin{array}{l}\text { Negative } \\
\text { Positive }\end{array}$ & 2.380 & $1.101-5.104$ & 0.026 \\
\hline M & $\begin{array}{l}\text { M0 } \\
\text { M1 }\end{array}$ & 4.092 & $1.371-12.210$ & 0.012 \\
\hline
\end{tabular}

$P$ : $P$ value; RR: relative risk to relapse; CI: confidence interval. Define $P<0.05$ as the significant difference. 


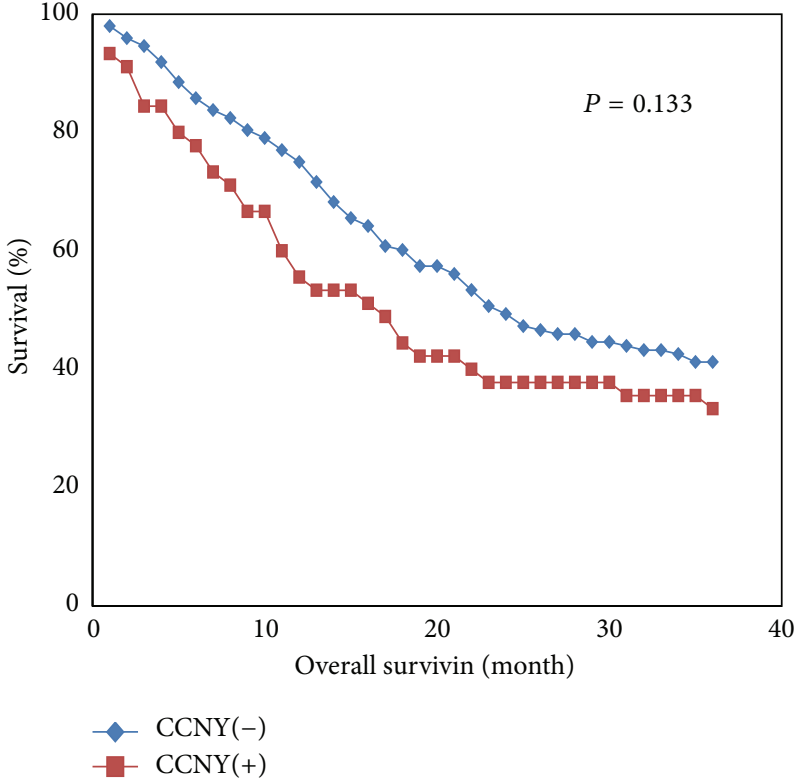

(a)

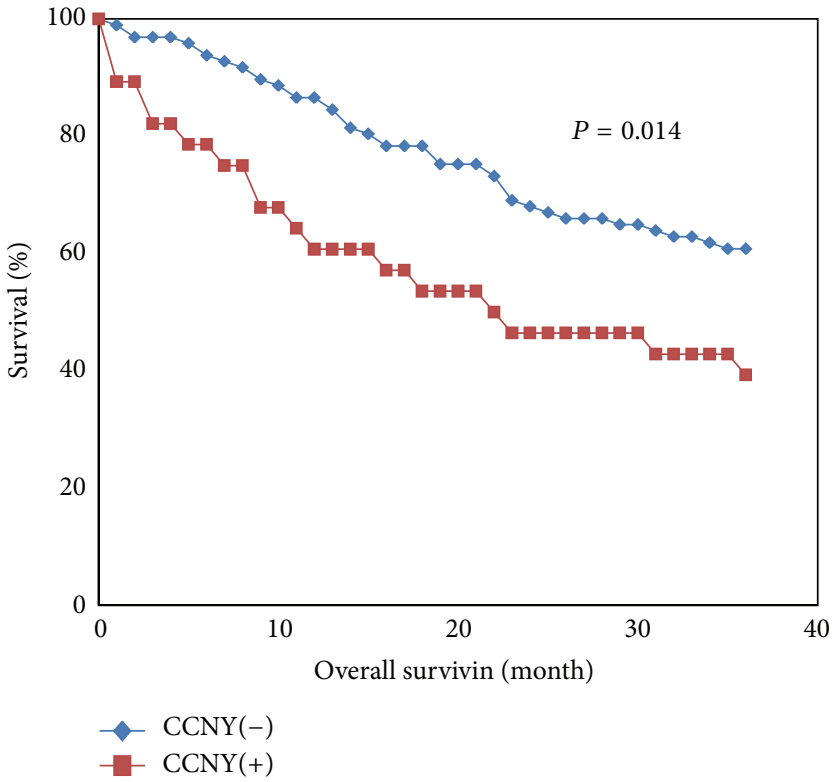

(b)

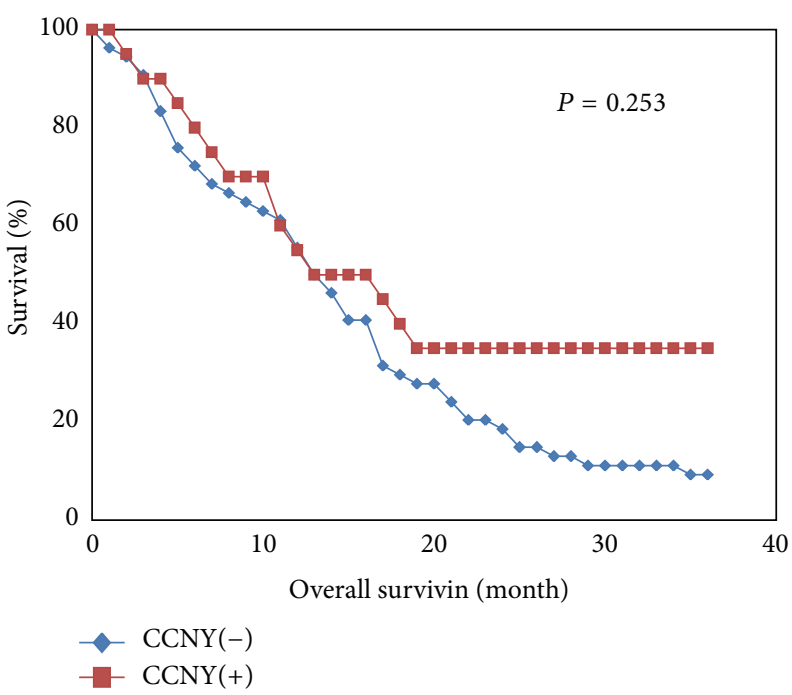

(c)

FIGURE 4: Kaplan-Meier survival curves for OS as determined by the levels of CCNY autoantibody. (a) All patients with nonsmall-cell lung cancer. (b) Postoperative patients with nonsmall cell lung cancer; (c) medical chemotherapy of patients with nonsmall cell-lung cancer.

is more likely to be related to the changes in protein levels. Serum autoantibody appears to be an early biomarker, as it has been described as being present before symptomatic disease [25-27]. Therefore, we further assessed the diagnostic value of anti-CCNY autoantibody using a ROC curve. The AUC was 0.737 (95\% CI 0.677-0.796), and the cutoff value of $112.5 \mathrm{ng} / \mathrm{mL}$ gave a sensitivity of $23.5 \%$ and specificity of 95.5\%. The serum levels of anti-CCNY autoantibody were also significantly higher in the patients of NSCLC than those of tuberculosis, but there was no significant difference in the positivity of serum anti-CCNY autoantibody between the patients of NSCLC and those of TB (23.5\% versus $19.3 \%$, $P=0.373)$. We also found no statistical significance on the levels of serum anti-CCNY autoantibody among the patients of early-stage NSCLC and patients of tuberculosis. Whether or not serum anti-CCNY autoantibody can be used as an early diagnosis or assistant diagnostic biomarker needs to be addressed by further research.

In addition, the present study demonstrates that antiCCNY autoantibody is associated with poor prognosis for NSCLC, compare with anti CCNY autoantibodies negative patients, the positive patients tended to have shorter survival times although the difference did not reach statistical significance. Currently, clinical stage is the most commonly used reference index of prognosis for patients with cancer. However, it does not take into account the effects of different treatments on patient prognosis. Surgical treatment is the most effective and preferred option for patients with early 
stage lung cancer. Chemotherapy is also an important therapeutic approach for patients with advanced lung cancer. To eliminate the influences of different therapeutic methods on patient survival, we assessed, respectively, the relationships between the levels of anti-CCNY autoantibodies and the OS rates for patients who received surgical treatment or medical chemotherapy. The OS of postoperative patients with earlystage NSCLC was significantly associated with the levels of serum anti-CCNY autoantibodies. However, for the NSCLC patient group who received chemotherapy, the OS values were not significantly different between the anti-CCNY autoantibody-positive group and anti-CCNY autoantibodynegative group. Therefore, serum anti-CCNY autoantibody appears to be a valuable indicator for postoperative patients with early-stage NSCLC. In addition, serum anti-CCNY autoantibody is probably an independent risk factor for postoperative patients with early stage NSCLC, as determined in the COX multivariate survival analysis. As a good indicator or method to evaluate postoperative survival is currently lacking, serum anti-CCNY autoantibody may have value in terms of monitoring the survival of postoperative patients, evaluating surgical curative effects and guiding the individualization of treatment.

In summary, although CCNY is the newest member of the cyclin family, no studies have assessed the clinical value of measuring CCNY protein and serum anti-CCNY autoantibody. This study is the first to show that CCNY protein is overexpressed in lung cancer tissues and that the serum antiCCNY autoantibody levels in patients with NSCLC are higher than in control patients. A limitation of the present study is that only patients with tuberculosis were included, and patients with other pulmonary benign diseases, for example, pneumonia, pulmonary emphysema, and bronchial asthma, were not involved. In addition, we did not have detailed clinical data to analyze the relationships between the levels of CCNY protein and the clinical parameters. The CCNY protein may be a useful reference marker for determining more malignant forms of cancer which is similar or superior to other members of cyclin family. And the serum antiCCNY autoantibody may be a useful marker for monitoring postoperative prognosis.

\section{Conflict of Interests}

The authors declare that there is no conflict of interests.

\section{Acknowledgments}

This study was supported by a Beijing Novel Program grant (no. 2006B34), the Beijing Double Ten Project (no. Z121107002812115), and Beijing Cultivation Project for Key Technical and Medicine Products (Z101100055610030) (all awarded to Wentao Yue).

\section{References}

[1] A. Jemal, R. C. Tiwari, T. Murray et al., "Cancer statistics, 2004," CA: A Cancer Journal for Clinicians, vol. 54, no. 1, pp. 8-29, 2004.
[2] A. Jemal, R. Siegel, J. Xu, and E. Ward, "Cancer statistics, 2010," CA: A Cancer Journal for Clinicians, vol. 60, no. 5, pp. 277-300, 2010.

[3] A. Jemal, F. Bray, M. M. Center, J. Ferlay, E. Ward, and D. Forman, "Global cancer statistics," CA: A Cancer Journal for Clinicians, vol. 61, no. 2, pp. 69-90, 2011.

[4] R. Siegel, D. Naishadham, and A. Jemal, "Cancer statistics, 2012," CA: A Cancer Journal for Clinicians, vol. 62, no. 1, pp. 10-29, 2012.

[5] R. Siegel, D. Naishadham, and A. Jemal, "Cancer statistics, 2013," CA: A Cancer Journal for Clinicians, vol. 63, no. 1, pp. 11-30, 2013.

[6] C. J. Sherr, “Cancer cell cycles," Science, vol. 274, no. 5293, pp. 1672-1677, 1996.

[7] A. Satyanarayana and P. Kaldis, "Mammalian cell-cycle regulation: several Cdks, numerous cyclins and diverse compensatory mechanisms," Oncogene, vol. 28, no. 33, pp. 2925-2939, 2009.

[8] D. Santamaria and S. Ortega, "Cyclins and CDKS in development and cancer: lessons from genetically modified mice," Frontiers in Bioscience, vol. 11, no. 1, pp. 1164-1188, 2006.

[9] U. Galderisi, F. P. Jori, and A. Giordano, "Cell cycle regulation and neural differentiation," Oncogene, vol. 22, no. 33, pp. 52085219, 2003.

[10] J. Jin, F. D. Smith, C. Stark et al., "Proteomic, functional, and domain-based analysis of in vivo 14-3-3 binding proteins involved in cytoskeletal regulation and cellular organization," Current Biology, vol. 14, no. 16, pp. 1436-1450, 2004.

[11] W. Yue, X. Zhao, L. Zhang et al., "Cell cycle protein cyclin Y is associated with human non-small-cell lung cancer proliferation and tumorigenesis," Clinical Lung Cancer, vol. 12, no. 1, pp. 4350, 2011.

[12] Y. Xu, Z. Wang, J. Wang, J. Li, H. Wang, and W. Yue, "Lentivirusmediated knockdown of cyclin Y (CCNY) inhibits glioma cell proliferation," Oncology Research, vol. 18, no. 8, pp. 359-364, 2010.

[13] L. Zhong, S. P. Coe, A. J. Stromberg, N. H. Khattar, J. R. Jett, and E. A. Hirschowitz, "Profiling tumor-associated antibodies for early detection of non-small cell lung cancer," Journal of Thoracic Oncology, vol. 1, no. 6, pp. 513-519, 2006.

[14] J. Y. Zhang, C. A. Casiano, X. X. Peng, J. A. Koziol, E. K. L. Chan, and E. M. Tan, "Enhancement of antibody detection in cancer using panel of recombinant tumor-associated antigens," Cancer Epidemiology Biomarkers and Prevention, vol. 12, no. 2, pp. 136143, 2003.

[15] S. J. Storr, J. Chakrabarti, A. Barnes, A. Murray, C. J. Chapman, and J. F. R. Robertson, "Use of autoantibodies in breast cancer screening and diagnosis," Expert Review of Anticancer Therapy, vol. 6, no. 8, pp. 1215-1223, 2006.

[16] G. Covini, E. K. L. Chan, M. Nishioka, S. A. Morshed, S. I. Reed, and E. M. Tan, "Immune response to cyclin B1 in hepatocellular carcinoma," Hepatology, vol. 25, no. 1, pp. 75-80, 1997.

[17] H. Suzuki, D. F. Graziano, J. McKolanis, and O. J. Finn, "T cell-dependent antibody responses against aberrantly expressed cyclin $\mathrm{B} 1$ protein in patients with cancer and premalignant disease," Clinical Cancer Research, vol. 11, no. 4, pp. 1521-1526, 2005.

[18] J. A. Koziol, J. Y. Zhang, C. A. Casiano et al., "Recursive partitioning as an approach to selection of immune markers for tumor diagnosis," Clinical Cancer Research, vol. 9, no. 14, pp. 5120-5126, 2003.

[19] H. Lu, V. Goodell, and M. L. Disis, "Humoral immunity directed against tumor-associated antigens as potential biomarkers for 
the early diagnosis of cancer," Journal of Proteome Research, vol. 7, no. 4, pp. 1388-1394, 2008.

[20] J. Y. Zhang, "Tumor-associated antigen arrays to enhance antibody detection for cancer diagnosis," Cancer Detection and Prevention, vol. 28, no. 2, pp. 114-118, 2004.

[21] F. D. Shi, J. Y. Zhang, D. Liu et al., "Preferential humoral immune response in prostate cancer to cellular proteins $\mathrm{p} 90$ and $\mathrm{p} 62$ in a panel of tumor-associated antigens," Prostate, vol. 63, no. 3, pp. 252-258, 2005.

[22] W. T. Yue, X. T. Zhao, L. N. Zhang et al., "Overexpression of cyclin $\mathrm{Y}$ in non-small cell lung cancer is associated with cancer cell proliferation," Science China Life Sciences, vol. 53, no. 4, pp. 511-516, 2010.

[23] J. Y. Zhang, R. Megliorino, X. X. Peng, E. M. Tan, Y. Chen, and E. K. L. Chan, "Antibody detection using tumor-associated antigen mini-array in immunodiagnosing human hepatocellular carcinoma," Journal of Hepatology, vol. 46, no. 1, pp. 107-114, 2007.

[24] J. Li, L. J. Wang, X. Ying et al., "Immunodiagnostic value of combined detection of autoantibodies to tumor-associated antigens as biomarkers in pancreatic cancer," Scandinavian Journal of Immunology, vol. 75, no. 3, pp. 342-349, 2012.

[25] F. Fernández Madrid, "Autoantibodies in breast cancer sera: candidate biomarkers and reporters of tumorigenesis," Cancer Letters, vol. 230, no. 2, pp. 187-198, 2005.

[26] Y. Li, A. Karjalainen, H. Koskinen et al., "p53 autoantibodies predict subsequent development of cancer," International Journal of Cancer, vol. 114, no. 1, pp. 157-160, 2005.

[27] X. Wang, J. Yu, A. Sreekumar et al., "Autoantibody signatures in prostate cancer," The New England Journal of Medicine, vol. 353, no. 12, pp. 1224-1235, 2005. 


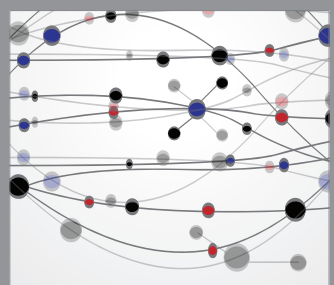

The Scientific World Journal
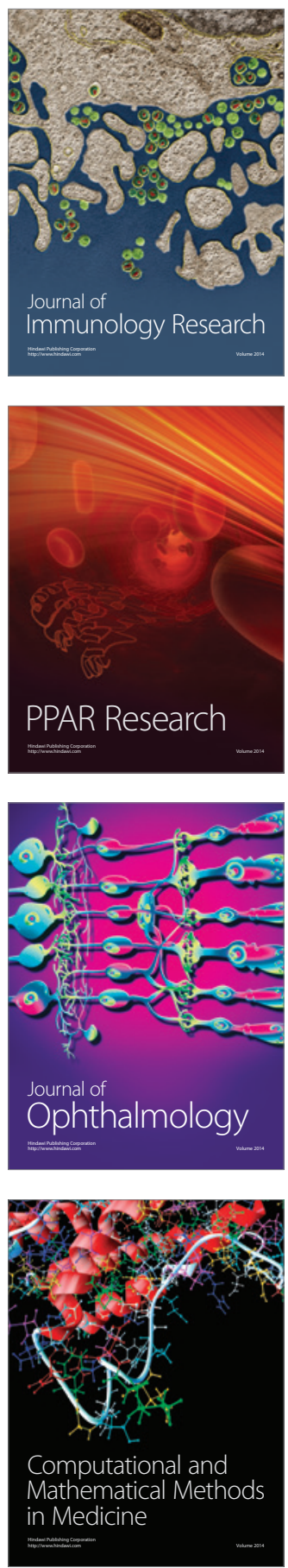

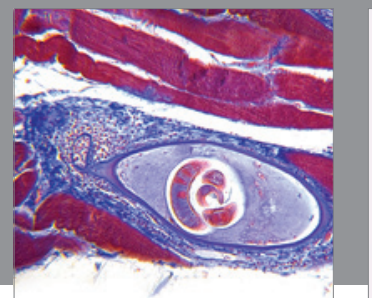

Gastroenterology

Research and Practice
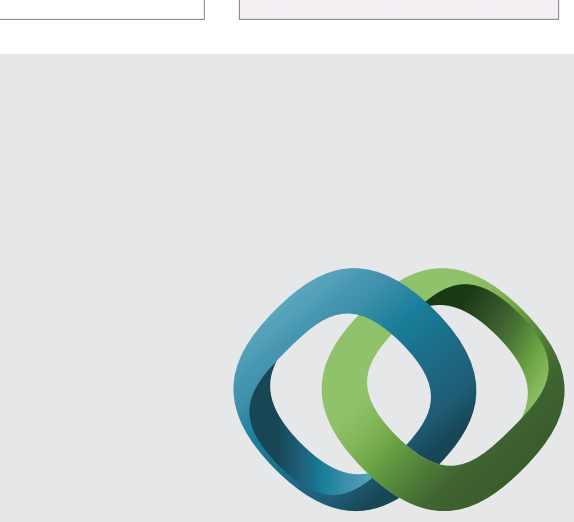

\section{Hindawi}

Submit your manuscripts at

http://www.hindawi.com
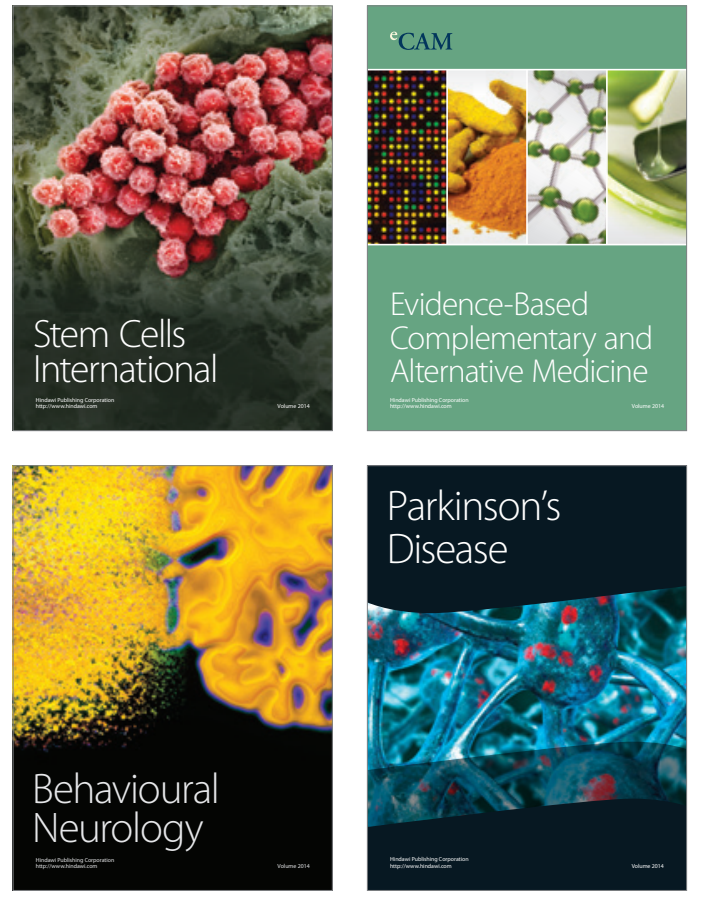
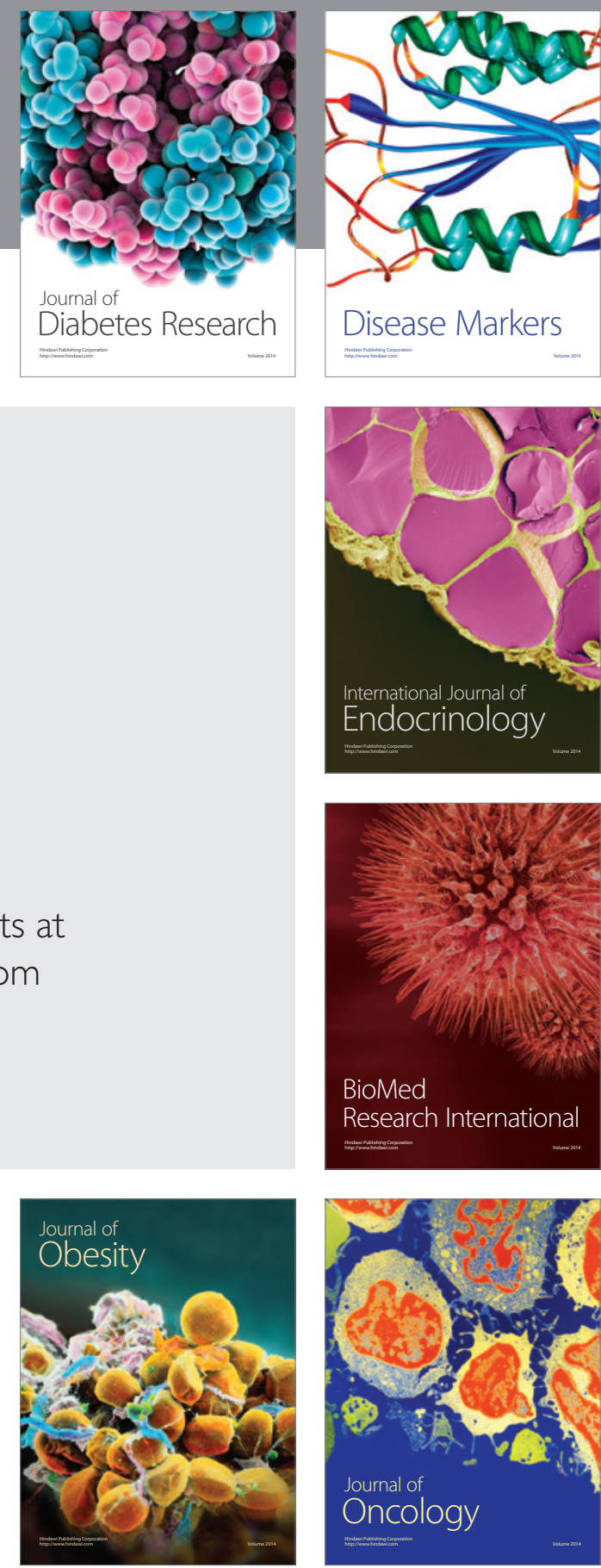

Disease Markers
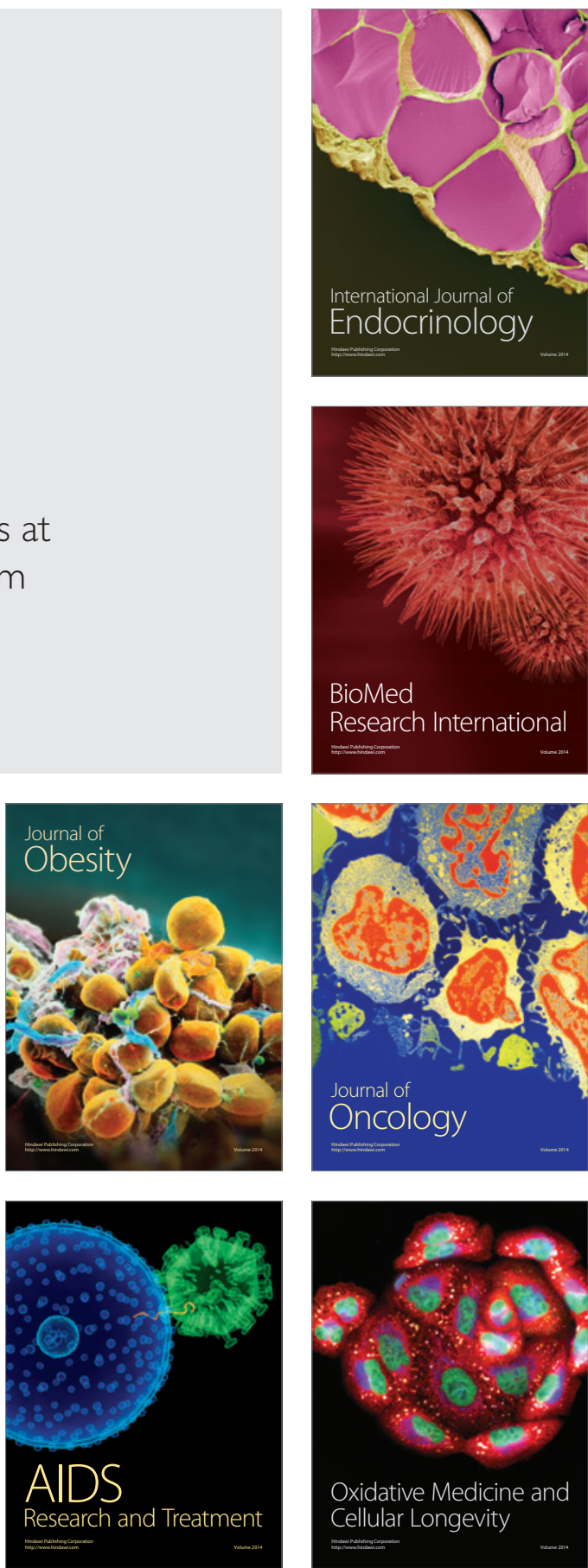